\title{
ON A CHARACTERISATION OF MATRIX FUNCTIONS WHICH ARE DIFFERENCES OF TWO MONOTONE MATRIX FUNCTIONS ${ }^{1}$
}

\begin{abstract}
HARKRISHAN VASUDEVA
Abstract. The class of matrix functions of 'bounded variation' was introduced by O. Dobsch in a paper published in 1937 [2]. The consideration of this class of functions immediately gives rise to the consideration of those matrix functions of order $n$ on an interval $[a, b]$ that are representable as the difference of two monotone matrix functions on that interval. Such a difference will have high regularity properties when $n$ is large and is therefore much more than simply a function of bounded variation. The characterization of this class was sought in the paper of Dobsch [2]. The purpose of this paper is to give a complete description of a related class: the functions defined on $(-1,1)$ which have restrictions to any closed subinterval which are such differences.
\end{abstract}

1. Introduction. This paper concerns the study of the class of matrix functions of 'bounded variation' corresponding to the monotone functions introduced by Charles Loewner in a paper published in 1934 [3]. O. Dobsch tried to find a characterisation of functions given on a closed interval $[a, b]$ which were the differences of monotone matrix functions on that interval. He did not succeed in finding this characterisation, and neither have we. However, we give a complete description of the functions on an open interval which appear on any closed subinterval as the differences of two monotone matrix functions on that subinterval.

2. If $A$ is an $n \times n$ real symmetric or Hermitian matrix, $f(A)$ is the matrix resulting from $A$ by leaving the eigenvectors fixed while the corresponding eigenvalues $\lambda$ are replaced by $f(\lambda)$. Thus, if $` A=T^{\prime} D T$ where $T$ is a unitary matrix, $T^{\prime}$ its conjugate transpose, and $D$ a diagonal matrix, then $F(A)=T^{\prime} f(D) T$. The function $f(A)$ on all $n \times n$ Hermitian matrices with eigenvalues in the domain of $f(x)$ is called a matrix function of order $n$ generated by $f$.

An operator function $f$ associated with $I$ is monotone provided

$$
H \geqq 0 \Rightarrow f(A+H) \geqq f(A) \text {. }
$$

Received by the editors June 8, 1970.

AMS 1970 subject classifications. Primary 26A45.

${ }^{1}$ This paper contains the results of the doctoral thesis of the author. This work was supported in part by National Science Foundation Grants GP 5436-1328.

(C) American Mathematical Society 1972 
When considering $n \times n$ Hermitian matrices, a monotone operator function is called a monotone matrix function of order $n$. A convenient summary of the results about monotone matrix functions may be found in [1]. Here we shall state a known result due to Dobsch [2].

THEOREM 1. Let $f(x)$ be a real-valued function defined on an open interval $(-1,1) .^{2} f(x)$ is a monotone matrix function of order $n$ in $(-1,1)$ iff $f$ is of class $C^{2 n-3}$, its $(2 n-3)$ rd derivative is convex and the matrix

$$
M_{n}^{\prime}(x ; f)=\left[\begin{array}{cccc}
f^{\prime}(x) & \frac{f^{\prime \prime}(x)}{2 !} & \cdots & \frac{f^{(n)}(x)}{n !} \\
\frac{f^{\prime \prime}(x)}{2 !} & \frac{f^{\prime \prime \prime}(x)}{3 !} & \frac{f^{(n+1)}(x)}{(n+1) !} \\
\cdot & \cdot & \cdot \\
\cdot & \cdot & \cdot \\
\frac{f^{(n)}(x)}{n !} & \frac{f^{(n+1)}(x)}{(n+1) !} & & \frac{f^{(2 n-1)}(x)}{(2 n-1) !}
\end{array}\right]
$$

(which makes sense almost everywhere) is nonnegative definite.

3. Definition. Let $f(x)$ be a real-valued function defined on a closed interval $[a, b] ; f(x)$ is called a matrix function of bounded variation if there exists a constant $K$ such that

$$
\sum_{v=1}^{p}\left\|f\left(A_{v}\right)-f\left(A_{v-1}\right)\right\| \leqq K
$$

for all partitions $a I \leqq A_{0} \leqq A_{1} \leqq \cdots \leqq A_{p} \leqq b I$.

Since the norms on the finite-dimensional space of matrices of order $n$ are equivalent, it is not necessary to specify exactly what norm occurs in the definition. For the special case $n=1$, it is clear that the matrix functions of bounded variation coincide with the usual functions of bounded variation over $[a, b]$. An important theorem due to Dobsch [2] asserts that this is also the case for larger values of $n$.

Below we give a complete description of a class: the functions defined on $(-1,1)$ whose restrictions to any closed subinterval generate matrix functions of order $n$ which are differences of two monotone matrix functions.

\footnotetext{
${ }^{2}$ Throughout this paper the restriction to the interval $(-1,1)$ is unessential. All results can easily be transformed to the case of an arbitrary open interval.
} 
Lemma 2. Let $C$ be a compact subset of the $n \times n$ Hermitian matrices such that every element of $C$ has its spectrum in $(-1,1)$. Let $h(x)=$ $\sqrt{ }(x+2)$; then there exists a $K>0$ so that the matrix

$$
A+K M_{n}^{\prime}(x ; h)
$$

is positive definite for every $A$ in $C$ and every $x$ in $(-1,1)$.

Proof. Let $\|A\|$ be a norm on the space of matrices which is at least as large as the usual operator norm so that the spectrum of $A$ is contained in the interval $(-\|A\|,\|A\|)$. This norm is clearly a continuous function on the compact $C$ and bounded there by some positive $\mu$. It follows that $-\mu I \leqq A \leqq \mu I$ for every $A$ in $C$.

Consider next the family of matrices $M_{n}^{\prime}(x ; h)$ as $x$ varies over the closed interval $[-1,1]$. This is evidently a compact set of matrices depending continuously on $x$. Every matrix in the set is positive and so there exists a positive $\alpha$ so that $M_{n}^{\prime}(x ; h) \geqq \alpha I$ for every $x$ in $(-1,1)$.

Note that if $\phi(x)=K h(x)$ then $A+M_{n}^{\prime}(x ; \phi)$ is positive definite for all $A$ in $C$ and all $x$ in $(-1,1)$.

THEOREM 3. A real-valued function $f(x)$ defined on $(-1,1)$ is the difference of two monotone matrix functions of order $n>1$ on every closed subinterval $[a, b]$ if and only if $f$ is $C^{2 n-3}$, the derivative $f^{(2 n-3)}(x)$ is absolutely continuous on $[a, b]$ and its derivative, $f^{(2 n-2)}(x)$ is of bounded variation there.

Proof. Let $[a, b]$ be an arbitrary but fixed closed subinterval of $(-1,1)$. Then there exists a closed subinterval $\left[a^{\prime}, b^{\prime}\right]$ of $(-1,1)$ such that $[a, b]$ is contained in the interior of $\left[a^{\prime}, b^{\prime}\right]$. If $f(x)$ is the difference of two monotone matrix functions of order $n>1$ on $\left[a^{\prime}, b^{\prime}\right]$, then in view of Theorem 1 , it follows that $f$ is $C^{2 n-3}$, the derivative $f^{(2 n-3)}(x)$ is absolutely continuous and its derivative, $f^{(2 n-2)}(x)$ is of bounded variation on at least $[a, b]$.

Suppose $f(x)$ is $C^{2 n-3}$, the derivative $f^{(2 n-3)}$ is absolutely continuous on $[a, b]$ and $f^{(2 n-2)}(x)$ is of bounded variation there. We shall show that $f$ can be expressed as the difference of two monotone functions of order $n$. Since $f^{(2 n-2)}$ is of bounded variation, it follows that $f=G_{1}-G_{2}$ where $G_{i}^{(2 n-2)}(i=1,2)$ are monotone increasing. The polynomial $p(x)$ which appears in the process of obtaining $f$ from $f^{(2 n-2)}$ may be absorbed into $G_{1}$. Hence it is enough to prove the theorem when $f^{(2 n-2)}$ is monotone increasing. To show that $f$ is the difference of two monotone matrix functions, we must find a convenient $h \in P(-1,1)^{3}$ so that the matrix

${ }^{3} P(-1,1)$ denotes the functions in the Pick class which are real and regular on the interval $(-1,1)$ and which therefore admit analytic continuation into the lower halfplane which is given by reflection. 
$M_{n}^{\prime}(x ; f+h)$ is a positive matrix for almost all $x \in[a, b]$. Setting $f(x)+h(x)=$ $g(x)$, we obtain $f(x)=g(x)-h(x)$ where $g(x)$ and $h(x)$ are such that $M_{n}^{\prime}(x ; g)$ and $M_{n}^{\prime}(x ; h)$ are positive matrices for almost all $x \in[a, b]$. By a theorem of Dobsch [1, Theorem 2.4] and remarks preceding Lemma 2 , it follows that $g(x)$ and $h(x)$ are monotone matrix functions of order $n$ on $[a, b]$. We write $M_{n}^{\prime}(x ; f)=A(x)+B(x)$ where

$$
B(x)=\left[\begin{array}{cccc}
0 & 0 & \ldots & 0 \\
0 & 0 & \ldots & 0 \\
\cdot & \cdot & & \cdot \\
\cdot & \cdot & & \cdot \\
\cdot & \cdot & & \cdot \\
0 & 0 & \ldots & \frac{f^{(2 n-1)}(x)}{(2 n-1) !}
\end{array}\right]
$$

and $A(x)$ is the remainder of $M_{n}^{\prime}(x ; f)$.

Since $A(x)$ does not contain $f^{(2 n-1)}(x)$, it is a uniformly bounded matrix function of $x$ in $[a, b]$. It follows from Lemma 2 that

$$
A(x)+\lambda M_{n}^{\prime}(x ; h)>0, \quad x \in[a, b],
$$

if $\lambda$ is sufficiently large. On the other hand $B(x) \geqq 0$ almost everywhere, because $f^{(2 n-1)}(x)>0$ almost everywhere. It follows that

$$
M_{n}^{\prime}(x ; f)+\lambda M_{n}^{\prime}(x ; h)>0
$$

almost everywhere, as required.

The author is indebted to the referee for simplifications of his original proof.

\section{REFERENCES}

1. J. Bendat and S. Sherman, Monotone and convex operator functions, Trans. Amer. Math. Soc. 79 (1955), 58-71. MR 18, 588.

2. O. Dobsch, Matrixfunktionen beschrankter Schwankung, Math. Z. 43 (1937), 353-388.

3. K. Loewner, Über monotone Matrixfunktionen, Math. Z. 38 (1934), 177-216.

Center for Advanced Study in Mathematics, Punjab University, Chandigarh 14, INDIA 